\title{
Nanotecnología: \\ El Futuro a un Nanómetro de Distancia
}

\author{
Mónica López Sardi *
}

\section{Resumen}

El prefijo nano se aplica para denominar los hallazgos vinculados a un campo de investigación nuevo, multidisciplinario y diverso que abre a la ciencia tantas posibilidades que parecen salidas de una historia de ciencia ficción. Desde nanorobots que circulen en nuestro torrente sanguíneo diagnosticando o curando enfermedades, hasta la promesa de una computadora superpotente del tamaño de una moneda de cinco centavos, todo parece al alcance de la mano gracias al futuro desarrollo de la nanotecnología.

Palabras clave: nanotecnología, nanomateriales, nuevas tecnologías.

\section{Abstract}

The nano prefix is applied to describe the findings related to a new research field. Also it is the name of a multidisciplinary science that brings possibilities that seems straight out from a science fiction story. Since nanorobots, that circulates in our bloodstream to diagnose or cure diseases, until the promise of a superpotent computer with the size of a nickel, everything seems posible thanks to the future development of nanotechnology.

Keywords: nanotechnology, nanomaterials, new technologies.

- Universidad de Palermo, Directora del Grupo de Investigación en Ingeniería Sustentable (GIIS) de la Facultad de Ingeniería. 


\section{Introducción}

El prefijo nano, que distingue todo lo relacionado con esta nueva disciplina, proviene del nanómetro $(\mathrm{nm})$, que es la unidad de longitud equivalente a la milmillonésima parte del metro $\left(1 \mathrm{~nm}=10^{-9} \mathrm{~m}\right)$.

La nanotecnología se considera un campo de las ciencias aplicadas, que estudia, diseña y sintetiza materiales, dispositivos y sistemas funcionales basándose en las propiedades de la materia a nanoescala. Estos dispositivos miden entre $1 \mathrm{y}$ $100 \mathrm{~nm}$. Un nanobot (o nanorobot) de $50 \mathrm{~nm}$ estaría formado por unas 5 capas de moléculas o átomos.

\section{Un poco de historia}

Richard Feynman, ganador del Premio Nobel de Física en 1965, es considerado el padre de la nanotecnología, dado que fue el primero que hizo mención a las posibilidades del nanomundo en la conferencia "En el fondo hay espacio de sobra", que dio en el CalTech en diciembre de 1959.

El avance de la ciencia a nanoescala fue posible a partir de 1980, con la aparición de la microscopía de efecto túnel y la microscopía de fuerza atómica. Esto hizo posible en primer lugar la observación y más adelante la manipulación de los materiales a escala atómica y molecular.

Los nanotubos de carbono (derivados de los fullerenos) son las partículas precursoras de la nanotecnología. Los fullerenos, descubiertos en 1985, son una de las formas más estables del carbono, tras el diamante y el grafito. En ellos los átomos del elemento se ordenan en una distribución de pentágonos y hexágonos, semejantes a un balón de fútbol. Fueron detectados por Kroto, Curl y Smalley (de la Universidad de Sussex) al someter al grafito a experimentos de espectroscopía molecular y aislados mediante una incisión con rayos láser. Por este descubrimiento recibieron el Nobel de Química en 1996. También se los ha detectado en el espacio, mediante el telescopio Spitzer, dado que son creados en las capas exteriores de las estrellas y expulsados al espacio durante sus explosiones. Se los puede sintetizar artificialmente, mediante síntesis química catalítica. Estas moléculas de carbono constituyen una de las bases primordiales de la nanotecnología.

Los fullerenos pueden portar gases en su interior, o se puede incrementar su reactividad mediante la unión de distintos grupos activos a su superficie. También pueden polimerizarse, dando lugar a la síntesis de los nanotubos de carbono. Estos nanotubos presentan propiedades excepcionales: son de 10 a 100 veces más fuertes que el acero por unidad de peso y pueden actuar como conductores o como semiconductores, dependiendo de la forma en que se enrollan. 


\section{Nanotecnología hoy}

En la actualidad, cientos de empresas privadas y laboratorios llevan el prefijo nano en su nombre, pero todavía existen pocos productos en el mercado.

Entre las aplicaciones que ya se encuentran en nuestra vida diaria podemos mencionar las nanocápsulas utilizadas en distintos cosméticos, cremas humectantes, antiarrugas y pantallas de protección solar con nanopartículas (como el dióxido de titanio) que absorben rayos UV. También existen nanomateriales usados para preservación y embalaje de alimentos. Se pueden comprar gafas y lentes con nanocapas imposibles de rayar. Ya existen materiales ligeros y fuertes para la defensa, la industria aeronáutica y automotriz. Se aplica nanotecnología para diseñar pantallas de TV y computadora más ligeras, finas y flexibles. También se aplica nanotecnología para obtener energía fotovoltaica en forma más eficiente, para pinturas y recubrimientos especiales, en sensores de óxido de cinc para los test de alcoholemia y hasta en el recubrimiento de pelotitas de golf, tenis y ping pong.

\section{Nanotecnología y futuro}

Grandes empresas del mundo informático y farmacéutico, así como distintos gobiernos, invierten cada año miles de millones de dólares en esta carrera tecnológica. Se espera que en un futuro cercano los avances en nanotecnología revolucionen el mundo y sean fuente de grandes negocios, como ha ocurrido con las tecnologías informáticas y las biotecnologías.

Las mayores esperanzas están cifradas en la nanomedicina. Ya los cambios se han hecho presentes en los productos dermatológicos, o en el incremento de la actividad y disminución de efectos indeseados de medicamentos tan tradicionales como la aspirina.

Para la lucha contra el cáncer se investiga un procedimiento que consiste en inyectar nanotubos de carbono en las células cancerosas, los cuales liberan calor al ser expuestos a un campo magnético, destruyendo la célula cancerosa. En otros casos los nanotubos transportan en su interior drogas para atacar tumores, que son liberadas hacia la célula al ser pulsadas por un campo electromagnético. Algunos de los estudios que se están realizando en este campo parecen provenir del terreno de la fantasía: los glóbulos rojos artificiales o "respirocitos", el micropáncreas artificial para la producción de insulina y los biochips capaces de detectar enfermedades infecciosas en sus estadios iniciales.

Del mismo modo se espera lograr avances espectaculares en los terrenos de la electrónica y la informática, el cuidado y limpieza del ambiente mediante 
nanodendritas metálicas y otras tecnologías, la industria textil y los tejidos inteligentes y tantos otros campos de aplicación.

\section{No todo lo que brilla es oro}

Los investigadores en este nuevo campo están abocados al desarrollo de tecnologías que, de concretarse, pueden cambiar la calidad y expectativas de vida de toda la humanidad. Pero dadas las reglas de juego del mercado, puede pasar mucho tiempo antes de que estos avances estén disponibles y a precios accesibles para la población en general.

Además, algunas voces comienzan a alzarse para advertir de los efectos negativos de las nanopartículas.

Ya en el 1997 investigadores de la Universidad de Oxford y la Universidad de Montreal demostraron que el dióxido de titanio y el óxido de zinc usados como nanopartículas en la mayoría de los protectores solares producen radicales libres en las células de la piel, produciendo daños en el ADN. Este mismo efecto se observó en cosméticos que usan nanopartículas, tales como las cremas antiarrugas y otros cosméticos de efecto rápido, convirtiéndose en una contradicción para la conservación de la salud. Las empresas más importantes de cosméticos reconocen estos efectos secundarios, y ya están recubriendo estas nanopartículas con nanocapas de otros materiales, para minimizar el problema.

Con respecto a la conservación de alimentos mediante nanomateriales protectores y embalajes que aplican estas tecnologías, en 2007, el Comité Mixto FAO/OMS de Expertos en Aditivos Alimentarios (JECFA) advirtió que ni las especificaciones ni las IDA (ingesta diaria admitida) correspondientes a las otras formas de aditivos alimentarios evaluadas podían extrapolarse sin más a las nanopartículas. Las futuras normas alimentarias deberán abordar por tanto de forma más concreta esas cuestiones.

Es importante que se desarrollen procedimientos para comprobar que estos productos innovadores cumplen la función que se atribuyen sin acarrear daños para el ambiente o la salud de las personas. Esto conlleva el nacimiento de una ciencia paralela a la nanotecnología: la nanotoxicología, que estudia la peligrosidad que estas partículas (muchas veces fotoactivas, con propiedades oxidantes, reductoras o catalíticas) pueden tener sobre los usuarios y los trabajadores de las plantas productoras. Ya se ha demostrado que el fullereno C60 provoca estrés oxidativo en peces y que ciertas nanopartículas al ser respiradas presentan el mismo efecto cancerígeno que el asbesto. 


\section{Conclusión}

Este camino recién comienza. Las predicciones de las películas "Viaje alucinante" de 1966 y "Querida, encogí a los niños" de 1989, empiezan a tomar un viso de realidad. La nanotecnología se encuentra en una etapa precompetitiva. De cumplirse las expectativas que genera esta nueva ciencia, el mundo del mañana, podría superar en mucho la fantasía propuesta por el cine.

\section{Fuentes}

ETICA Y NANOTECNOLOGÍAS EN SALUD. Juan Carlos Tealdi. Director del Programa de Bioética del Hospital de Clínicas de la Universidad de Buenos Aires.

Richard P. Feynman. "There's Plenty of Room at the Bottom" (conference). American Physical Society at CalTech., December 29 $9^{\text {th }}, 1959$.

http://revista.consumer.es/web/es/20041101/medioambiente/

www.portalciencia.net/nanotecno/

http://www.euroresidentes.com/futuro/nanotecnologia/diccionario/nanotubos.htm http://www.invdes.com.mx/suplemento-noticias/1564-nanotecnologia-paralimpiar-el-medio-ambiente 
